Appl. Set-Valued Anal. Optim. 4 (2022), No. 1, pp. 55-71

Available online at http://asvao.biemdas.com

https://doi.org/10.23952/asvao.4.2022.1.04

\title{
A MODIFIED INERTIAL PROJECTION AND CONTRACTION METHOD FOR SOLVING BILEVEL SPLIT VARIATIONAL INEQUALITY PROBLEMS
}

\author{
G.C. UGWUNNADI ${ }^{1,2}$, C. IZUCHUKWU ${ }^{3, *}$, L.O. JOLAOSO ${ }^{2,4}$, C.C OKEKE ${ }^{5}$, K.O. AREMU ${ }^{2,6}$ \\ ${ }^{1}$ Department of Mathematics, University of Eswatini, Kwaluseni, Eswatini \\ ${ }^{2}$ Department of Mathematics and Applied Mathematics, \\ Sefako Makgatho Health Sciences University, Ga-Rankuwa, Pretoria, South Africa \\ ${ }^{3}$ Department of Mathematics, The Technion-Israel Institute of Technology, Haifa, Israel \\ ${ }^{4}$ Department of Mathematics, College of Physical Sciences, \\ Federal University of Agriculture, Abeokuta, Ogun State, Nigeria \\ ${ }^{5}$ School of Mathematics, University of the Witwatersrand, Private Bag 3, Johannesburg 2050, South Africa \\ ${ }^{6}$ Department of Mathematics, Usmanu Danfodiyo University Sokoto, Sokoto State, Nigeria
}

\begin{abstract}
The main purpose of this paper is to study a bilevel split variational inequality problem in two real Hilbert spaces. We propose a new modified inertial projection and contraction method for solving this problem when one of the cost operators is pseudomonotone and Lipschitz continuous, but not sequentially weakly continuous. Strong convergence of the proposed method is established and numerical examples are given to support our theoretical findings.
\end{abstract}

Keywords. Bilevel split variational inequality problem; Generalized demimetric mapping; Pseudomonotone operator; Projection and contraction method.

\section{INTRODUCTION}

Let $H_{1}$ and $H_{2}$ be real Hilbert spaces endowed with $\langle.,$.$\rangle and norm \|\cdot\|$. Let $C$ and $Q$ be nonempty, closed and convex subsets of real Hilbert spaces $H_{1}$ and $H_{2}$, respectively. Let $B$ : $H_{1} \rightarrow H_{2}$ be a bounded linear operators, and let $A, F_{1}: H_{1} \rightarrow H_{1}$ and $F_{2}: H_{2} \rightarrow H_{2}$ be nonlinear mappings. The bilevel split variational inequality problem (BSVIP) is defined as follows:

$$
\text { Finding } z^{*} \in \Gamma \text { such that }\left\langle F_{1}\left(z^{*}\right), z-z^{*}\right\rangle \geq 0, \forall z \in \Gamma \text {, }
$$

where $\Gamma:=\left\{z^{*} \in V I(C, A): B z^{*} \in V I\left(Q, F_{2}\right)\right\}$ is the solution set of the following split variational inequality problem (SVIP) introduced by Censor et al. [1]:

$$
\text { Finding } z^{*} \in C \text { solves }\left\langle A\left(z^{*}\right), u-z^{*}\right\rangle \geq 0, \forall u \in C
$$

such that

$$
x^{*}=B z^{*} \in Q \text { solves }\left\langle F_{2}\left(x^{*}\right), y-x^{*}\right\rangle \geq 0, \forall y \in Q,
$$

* Corresponding author.

E-mail addresses: ugwunnadi4u@yahoo.com (G.C. Ugwunnadi), izuchukwu_c@yahoo.com; chi.izuchukw @ campus.technion.ac.il (C. Izuchukwu), lateef.jolaoso@smu.ac.za (L.O.Jolaoso), chibueze.okeke87@yahoo.com (C.C. Okeke), aremukazeemolalekan@gmail.com (K.O. Aremu).

Received April 14, 2021; Accepted May 3, 2021.

(C)2022 Applied Set-Valued Analysis and Optimization 
and $V I(C, A)$ and $V I\left(Q, F_{1}\right)$ denote the solution sets of variational inequalities (1.2) and (1.3), respectively. Censor et al. [1] proposed and studied the following method for solving the SVIP (1.2)-(1.3) as follows: For $x_{1} \in H_{1}$,

$$
x_{n+1}=P_{C}(I-\lambda A)\left(x_{n}+\tau B^{*}\left(P_{Q}\left(I-\lambda F_{2}\right)-I\right) B x_{n}\right), n \geq 1,
$$

where $A$ and $F_{2}$ are $\beta_{1}$ and $\beta_{2}$-inverse strongly monotone, and $\lambda$ and $\tau$ satisfy some conditions. They proved weak convergence of the sequence generated by (1.4) to a solution of problem (1.2)-(1.3). The SVIP (1.2)-(1.3) is a very interesting union of classical variational inequality problem (1.2), which was first introduced by Stampacchia [2] and have been used as a strong methodology in studying traffic equilibrium control problems, machine learning, medical imaging, see, e.g., $[3,4,5]$ for more details. A special case of the SVIP, when $A=F_{2}=0$, is the split feasibility problem (SFP) introduced and studied by Censor and Elfving [6], which has been studied and applied in many fields such as phase retrieval, medical image reconstruction, signal processing, radiation therapy treatment planning, and so on; see, e.g, [7, 8, 9] for more details. It is also known that problem (1.2) is equivalent to the fixed point problem:

$$
\text { Finding } z^{*} \in C \text { such that } z^{*}=P_{C}\left(z^{*}-\lambda A z^{*}\right) \text {, }
$$

where $\lambda>0$ and $P_{C}$ is the metric projection of $H_{1}$ onto $C$. Many methods have been developed for solving the VIP (1.2). One of the methods is the extragradient method which was proposed and studied by Korpelevich [10] and Antipin [11] in finite dimensional Euclidean spaces as follows:

$$
x_{1} \in C, y_{n}=P_{C}\left(x_{n}-\lambda A x_{n}\right), x_{n+1}=P_{C}\left(x_{n}-\lambda A y_{n}\right), \forall n \geq 1 \text {, }
$$

where $A$ is a monotone and $L$-Lipschitz continuous operator. Under the assumption that $V I(C, A)$ is nonempty, the sequence generated by (1.6) converges to a point in $\operatorname{VI}(C, A)$. This extragradient method may be costly in computation, since it requires two orthogonal projections at each iteration. There are some methods to overcome this difficulty, one of which is the subgradient extragradient method introduced by Censor et al. [12], where the second projection onto the constrained set is replaced with a projection onto a specific constructible half-space. Another method, which was proposed by Tseng [13], is called the Tseng's extragradient method. The method only requires one projection onto the feasible set. Since the subgradient extragradient method and Tseng's extragradient methods requires only one projection onto the constrained set in each iteration, they have attracted the attention of many researchers working in this field; see, e.g., $[14,15,16,17]$ and the references therein. However, there is also a setback in terms of the applicability of subgradient extragradient method and Tseng's extragradient method which is the fact that the stepsize depends on the knowledge of the Lipschitz constant. The third method is the projection and contraction method (PCM), proposed and studied by He [18] (see also Sun [19]):

$$
\left\{\begin{array}{l}
x_{1} \in H \\
y_{n}=P_{C}\left(x_{n}-\lambda A x_{n}\right), \\
d\left(x_{n}, y_{n}\right)=\left(x_{n}-y_{n}\right)-\lambda\left(A x_{n}-A y_{n}\right), \\
x_{n+1}=x_{n}-\gamma \eta_{n} d\left(x_{n}, y_{n}\right), \forall n \geq 1
\end{array}\right.
$$


where $\gamma \in(0,2), \lambda \in(0,1 / L)$ and $\eta_{n}:=\frac{\left\langle x_{n}-y_{n}, d\left(x_{n}, y_{n}\right)\right\rangle}{\left\|d\left(x_{n}, y_{n}\right)\right\|^{2}}$. He [18] established that the sequence $\left\{x_{n}\right\}$ generated by (1.7) converges weakly to a solution of (1.2). Since PCM requires only one projection onto the feasible set $C$, it reduces the computational cost per each iteration. This attracted the attention of many researchers who devoted their studies to improving the PCM in many different ways; see, e.g., [20, 21, 22] and the references therein. To speed up convergence rate of algorithms, Polyak [23] studied the heavy ball method, an inertial extrapolation process for minimizing a smooth convex function. Since then, many authors have introduced this technique in different methods for solving VIPs (see, e.g., [24, 25, 26, 27, 28] for details). In particular, Cholamjiak et al. [28] introduced the following inertial PCM for solving the VIP with pseudomonotone operator:

$$
\left\{\begin{array}{l}
x_{0}, x_{1} \in H, \\
w_{n}=x_{n}+\theta_{n}\left(x_{n}-x_{n-1}\right), \\
y_{n}=P_{C}\left(w_{n}-\lambda A w_{n}\right), \\
z_{n}=w_{n}-\gamma \eta_{n} d\left(w_{n}, y_{n}\right), \\
d\left(w_{n}, y_{n}\right)=\left(w_{n}-y_{n}\right)-\lambda\left(A w_{n}-A y_{n}\right), \\
\eta_{n}:=\frac{\left\langle w_{n}-y_{n}, d\left(w_{n}, y_{n}\right)\right\rangle}{\left\|d\left(w_{n}, y_{n}\right)\right\|^{2}}, \\
x_{n+1}=\left(1-\alpha_{n}-\delta_{n}\right) x_{n}+\alpha_{n} z_{n}, \forall n \geq 1,
\end{array}\right.
$$

where $A$ is $L$-Lipschitz continuous, pseudomonotone, and sequentially weakly continuous, $\gamma \in$ $(0,2), \lambda \in(0,1 / L),\left\{\tau_{n}\right\} \subset(0, \infty), \tau_{n}=\circ\left(\alpha_{n}\right),\left\{\alpha_{n}\right\} \subset\left(a, 1-\delta_{n}\right)$ for some $a>0,\left\{\delta_{n}\right\} \subset$ $(0,1), \theta>0$ and $\theta_{n}$ is selected such that $0 \leq \theta_{n} \leq \bar{\theta}_{n}$ and $\bar{\theta}_{n}:=\min \left\{\theta, \frac{\tau_{n}}{\left\|x_{n}-x_{n-1}\right\|}\right\}$ if $x_{n} \neq x_{n-1}$; otherwise, $\bar{\theta}_{n}=\theta$. They proved that the sequence $\left\{x_{n}\right\}$ generated by (1.8) converges strongly to the solution of the VIP. We note that Algorithm (1.8) requires to know the Lipschitz constant of operator $A$, which is often unknown or difficult to estimate in practice.

A special case of the BSVIP (1.1), when $H_{1}=H_{2}, F_{2}=0$ and $B=I$ (identity mapping), is known as a bilevel variational inequality problem (BVIP). The BVIP covers many aspects of mathematical programs with equilibrium constraints [29], bilevel convex programming models [30], and the minimum-norm problems with the solution set of variational inequalities [31]. Using the projection and contraction method, Thong et al. [32] studied the BVIP as follows:

$$
\left\{\begin{array}{l}
x_{1} \in H, \\
y_{n}=P_{C}\left(x_{n}-\lambda_{n} A x_{n}\right), \\
z_{n}=x_{n}-\alpha \eta_{n} d\left(x_{n}, y_{n}\right), \\
d\left(x_{n}, y_{n}\right)=\left(x_{n}-y_{n}\right)-\lambda_{n}\left(A x_{n}-A y_{n}\right), \\
\eta_{n}:=\frac{\left\langle x_{n}-y_{n}, d\left(x_{n}, y_{n}\right)\right\rangle}{\left\|d\left(x_{n}, y_{n}\right)\right\|^{2}}, \\
x_{n+1}=z_{n}-\alpha_{n} \gamma F\left(z_{n}\right), \forall n \geq 1,
\end{array}\right.
$$

where $A$ is $L_{1}$-Lipschitz continuous, pseudomonotone, and sequentially weakly continuous, $F$ is $\beta$-strongly monotone and $L_{2}$ - Lipschitz continuous, $\alpha \in(0,2), \mu \in(0,1), \lambda_{0}>0,0<\gamma<\frac{2 \beta}{L_{2}^{2}}$, $\left\{\alpha_{n}\right\} \subset(0,1)$ such that $\lim _{n \rightarrow \infty} \alpha_{n}=0, \sum_{n=1}^{\infty} \alpha_{n}=\infty$ and $\lambda_{n+1}:=\min \left\{\lambda_{n}, \mu \frac{\left\|x_{n}-y_{n}\right\|}{\left\|A x_{n}-A y_{n}\right\|}\right\}$ if $A x_{n} \neq A y_{n}$; otherwise, $\lambda_{n+1}=\lambda_{n}$. They proved that the sequence $\left\{x_{n}\right\}$ generated by (1.9) converges strongly to the unique solution of the BVIP. Very recently, Tan et al. [33] proposed the following inertial subgradient extragradient method and inertial Tseng's method, respectively, 
for solving the BVIP:

$$
\left\{\begin{array}{l}
x_{0}, x_{1} \in H \\
w_{n}=x_{n}+\theta_{n}\left(x_{n}-x_{n-1}\right) \\
y_{n}=P_{C}\left(w_{n}-\lambda_{n} A w_{n}\right) \\
T_{n}=\left\{x \in H:\left\langle w_{n}-\lambda_{n} A w_{n}-y_{n}, x-y_{n}\right\rangle \leq 0\right\} \\
z_{n}=P_{T_{n}}\left(w_{n}-\lambda_{n} A y_{n}\right) \\
x_{n+1}=z_{n}-\alpha_{n} \gamma F\left(z_{n}\right), \forall n \geq 1
\end{array}\right.
$$

and

$$
\left\{\begin{array}{l}
x_{0}, x_{1} \in H \\
w_{n}=x_{n}+\theta_{n}\left(x_{n}-x_{n-1}\right), \\
y_{n}=P_{C}\left(w_{n}-\lambda_{n} A w_{n}\right), \\
z_{n}=y_{n}-\lambda_{n}\left(A y_{n}-A w_{n}\right), \\
x_{n+1}=z_{n}-\alpha_{n} \gamma F\left(z_{n}\right), \forall n \geq 1 .
\end{array}\right.
$$

Under some assumptions on the parameters, they proved that Algorithms (1.10) and (1.11) converge strongly to the unique solution of the BVIP when $A$ is $L_{1}$-Lipschitz continuous, pseudomonotone and sequentially weakly continuous, and $F$ is $\beta$-strongly monotone and $L_{2}$ Lipschitz-continuous.

Motivated by the above works and using the idea presented in (1.5), letting $S(x):=P_{Q}(x-$ $\left.\lambda F_{2}(x)\right), \lambda>0$, we obtain that $\operatorname{VI}\left(Q, F_{2}\right)=F i x(S)(F i x(S)$ denotes the fixed points set of $S)$. In this case, we redefine BSVIP (1.1) as follows: Suppose that $A: H_{1} \rightarrow H_{1}$ is pseudomonotone and $K$-Lipschitz continuous (not sequentially weakly continuous), $F: H_{1} \rightarrow H_{1}$ is $\beta$-strongly monotone and $L$-Lipschitz continuous, $B: H_{1} \rightarrow H_{2}$ is a bounded linear operator with $B \neq 0$, and $S: H_{2} \rightarrow H_{2}$ is a $\kappa$-generalized demimetric mapping with $\kappa>0$. Our goal is to study the following BSVIP:

$$
\text { Finding } z^{*} \in \Gamma \text { such that }\left\langle F\left(z^{*}\right), z-z^{*}\right\rangle \geq 0, \forall z \in \Gamma \text {, }
$$

where $\Gamma:=\left\{z^{*} \in V I(C, A): B z^{*} \in F i x(S)\right\}$. We then propose a modified projection and contraction method with inertial extrapolation steps, and prove that the sequence generated by this algorithm converges strongly to a unique solution of BSVIP (1.12). We also give some numerical examples to show the efficiency of our proposed method.

\section{PRELIMinaries}

Let $C$ be a closed convex subset of a real Hilbert space $H$. Then, for each $x \in H$, there exists a unique point $z=P_{C}(x)$ such that $\|x-z\|=\inf _{y \in C}\|x-y\|$. The operator $P_{C}: H \rightarrow C$ is called the metric projection from $H$ onto $C$. We have that $z=P_{C}(x)$ if and only if the following relation holds $\langle x-z, y-z\rangle \leq 0, \forall y \in C$.

Recall that an operator $A: H \rightarrow H$ is said to be:

(a) $L$-Lipschitz continuous with $L>0$ if

$$
\|A x-A y\| \leq L\|x-y\|, \forall x, y \in H ;
$$


(b) $\beta$-strongly monotone if there exists $\beta>0$ such that

$$
\langle A x-A y, x-y\rangle \geq \beta\|x-y\|^{2}, \forall x, y \in H ;
$$

(c) monotone if

$$
\langle A x-A y, x-y\rangle \geq 0, \forall x, y \in H
$$

(d) pseudomonotone if

$$
\langle A x, y-x\rangle \geq 0 \Rightarrow\langle A y, y-x\rangle \geq 0, \forall x, y \in H .
$$

It is clear that $(b) \Rightarrow(c) \Rightarrow(d)$, but the converses are not true in general. Recall that a mapping $S: C \rightarrow X$ with $\operatorname{Fix}(S) \neq \emptyset$ is said to be $\kappa$-generalized demimetric if there exists $\kappa \in \mathbb{R} \backslash\{0\}$ such that $\|x-S x\|^{2} \leq \kappa\langle x-u, x-S x\rangle$, for all $x \in C$ and $u \in F i x(S)$. Let $S: C \rightarrow X$ be a $\theta$-generalized demimetric mapping with $\theta \in \mathbb{R} \backslash\{0\}$. Then $F(T)$ is closed and convex; see, [34].

The following lemma is trivial.

Lemma 2.1. Let $H$ be a real Hilbert space. Then, for all $x, y \in H$ and $\alpha \in \mathbb{R}$, the following hold

(i) $\|x-y\|^{2}=\|x\|^{2}+\|y\|^{2}-2\langle x, y\rangle$,

(ii) $\|x+y\|^{2} \leq\|x\|^{2}+2\langle y, x+y\rangle$,

(iii) $\|\alpha x+(1-\alpha) y\|^{2}=\alpha\|x\|^{2}+(1-\alpha)\|y\|^{2}-\alpha(1-\alpha)\|x-y\|^{2}$.

Lemma 2.2. [35] Let $H$ be a real Hilbert space, and let $F: H \rightarrow H$ be a $\beta$-strongly monotone and L-Lipschitz continuous mapping on $H$. If $\alpha \in(0,1), \eta \in[0,1-\alpha]$ and $\mu \in\left(0, \frac{2 \beta}{L^{2}}\right)$, then, for all $x, y \in H,\|[(1-\eta) x-\alpha \mu F(x)]-[(1-\eta) y-\alpha \mu F(y)]\| \leq(1-\eta-\alpha \tau)\|x-y\|$, where $\tau=1-\sqrt{1-\mu\left(2 \beta-\mu L^{2}\right)} \in(0,1)$.

Lemma 2.3. [36] Let $C$ be a nonempty closed and convex subset of a real Hilbert space $H$, and let $A: C \rightarrow H$ be a pseudomonotone and continuous operator. Then, $x^{*} \in \operatorname{VI}(C, A) \Leftrightarrow$ $\left\langle A(z), z-x^{*}\right\rangle \geq 0, \forall z \in C$.

Lemma 2.4. [37] Let $\left\{a_{n}\right\}$ be a nonnegative sequence of real numbers, $\left\{r_{n}\right\}$, and $\left\{s_{n}\right\}$ be sequences of real numbers such that $\left\{r_{n}\right\}$ is in $(0,1)$ with condition $\sum_{n=1}^{\infty} r_{n}=\infty$. Assume that $a_{n+1} \leq\left(1-r_{n}\right) a_{n}+r_{n} s_{n}, \forall n \geq 1$. If $\limsup _{k \rightarrow \infty} s_{n_{k}} \leq 0$ for every subsequence $\left\{a_{n_{k}}\right\}$ of $\left\{a_{n}\right\}$ satisfying the following condition: $\liminf _{k \rightarrow \infty}\left(a_{n_{k}+1}-a_{n_{k}}\right) \geq 0$, then $\lim _{n \rightarrow \infty} a_{n}=0$.

\section{MAin Results}

In this section, we introduce our method for solving the bilevel split variational inequality problem. We begin with the following assumptions.

Assumption 3.1. Let $H_{1}$ and $H_{2}$ be two real Hilbert spaces. Suppose the following conditions are satisfied:

(A1) the feasible set $C$ is a nonempty closed, and convex subset of $H_{1}$, and the solution set $\Gamma \neq \emptyset$

(A2) $A: H_{1} \rightarrow H_{1}$ is pseudomonotone, and $K$-Lipschitz continuous on $H_{1}$ with $K>0$;

(A3) $A: H_{1} \rightarrow H_{1}$ satisfies the following condition whenever $\left\{x_{n}\right\} \subset C, x_{n} \rightarrow z, \quad$ one has $\quad\|A z\| \leq \liminf _{n \rightarrow \infty}\left\|A x_{n}\right\|$; 
(A4) $F: H_{1} \rightarrow H_{1}$ is $\beta$-strongly monotone and $L$-Lipschitz continuous on $H_{1}$ with $L>0$ such that $\delta=1-\sqrt{1-\sigma\left(2 \beta-\sigma L^{2}\right)}$, where $\sigma \in\left(0, \frac{2 \beta}{L^{2}}\right)$;

(A5) $B: H_{1} \rightarrow H_{2}$ is a bounded linear operator such that $B \neq 0$ and $S: H_{2} \rightarrow H_{2}$ is $\kappa$ generalized demimetric with $\kappa \in(0, \infty)$ such that $S$ is demiclosed at the origin;

(A6) $\left\{c_{n}\right\}$ is a positive sequence with $c_{n}=\circ\left(b_{n}\right),\left\{a_{n}\right\} \subset\left(b, 1-b_{n}\right)$ for some $b>0,\left\{\lambda_{n}\right\}$ satisfies $0<c \leq \lambda \leq d<\frac{2}{\kappa \mid\|B\|^{2}}$ and $\left\{b_{n}\right\} \subset(0,1)$ satisfies $\lim _{n \rightarrow \infty} b_{n}=0$ and $\sum_{n=1}^{\infty} b_{n}=\infty$.

We remark here that Assumption (A3) is strictly weaker than the sequentially weakly continuous assumption which has frequently been used i for solving pseudomonotone VIPs recently. An example of an operator satisfying condition (A3) but not sequentially weakly continuous is $A(x)=x\|x\|$ (see [38, Remark 3.2]). Let $x^{*} \in H_{1}$ denote the unique solution of the BSVIP (1.12). We present the following modified inertial projection and contraction method for solving BSVIP (1.12).

Algorithm 3.1. Initialization: Choose $\alpha \in(0,2), \tau_{1}>0, \mu \in(0,1)$, and $\theta \in[0,1)$, and let $x_{0}, x_{1} \in H_{1}$ be arbitrary.

Iterative Steps: Calculate $x_{n+1}$ as follows:

Step 1. Given the iterates $x_{n-1}$ and $x_{n}$ for each $n \geq 1$, choose $\theta_{n}$ such that $0 \leq \theta_{n} \leq \bar{\theta}_{n}$, where

$$
\bar{\theta}_{n}=\min \left\{\theta, \frac{c_{n}}{\left\|x_{n}-x_{n-1}\right\|}\right\} \text {, if } x_{n} \neq x_{n-1} \text {; otherwise, set } \bar{\theta}_{n}=\theta \text {. }
$$

Step 2. Set

$$
y_{n}=x_{n}+\theta_{n}\left(x_{n}-x_{n-1}\right)
$$

and compute

$$
w_{n}=P_{C}\left(y_{n}-\tau_{n} A y_{n}\right)
$$

Update

$$
\tau_{n+1}= \begin{cases}\min \left\{\frac{\mu|| y_{n}-w_{n} \|}{\left\|A y_{n}-A w_{n}\right\|}, \tau_{n}\right\}, & \text { if } A y_{n} \neq A w_{n} \\ \tau_{n}, & \text { Otherwise }\end{cases}
$$

Step 3. Compute

$$
v_{n}=y_{n}-\alpha \eta_{n} d_{n}
$$

where

$d_{n}:=y_{n}-w_{n}-\tau_{n}\left(A y_{n}-A w_{n}\right), \eta_{n}=\frac{\left\langle y_{n}-w_{n}, d_{n}\right\rangle}{\left\|d_{n}\right\|^{2}}$, if $d_{n} \neq 0$; otherwise $\eta_{n}=0$.

Step 4. Compute

$$
\begin{aligned}
& u_{n}=v_{n}-\lambda_{n} B^{*}(I-S) B v_{n} \\
& x_{n+1}=a_{n} x_{n}+\left(1-a_{n}\right) u_{n}-b_{n} \sigma F u_{n} .
\end{aligned}
$$

Set $n:=n+1$ and return to Step 1 .

Lemma 3.1. ([39, Lemma 3.1]) The sequence $\left\{\tau_{n}\right\}$ generated by Algorithm 3.1 is non-increasing and

$$
\lim _{n \rightarrow \infty} \tau_{n}=\tau \geq \min \left\{\frac{\mu}{K}, \tau_{1}\right\}
$$


Lemma 3.2. Let $\left\{x_{n}\right\}$ be a sequence generated by Algorithm 3.1 under Assumption 3.1. Then $\left\{x_{n}\right\}$ is bounded.

Proof. Let $x^{*} \in \Gamma$. Since $w_{n} \in C$, we have $\left\langle A x^{*}, w_{n}-x^{*}\right\rangle \geq 0$, which implies by the pseudomonotonicity of $A$ that $\left\langle A w_{n}, w_{n}-x^{*}\right\rangle \geq 0$. Note that

$$
\left\langle y_{n}-w_{n}-\tau_{n} A y_{n}, w_{n}-x^{*}\right\rangle \geq 0 .
$$

It follows that $\left\langle y_{n}-w_{n}-\tau_{n}\left(A y_{n}-A w_{n}\right), w_{n}-x^{*}\right\rangle \geq 0$. Thus, $\left\langle w_{n}-x^{*}, d_{n}\right\rangle \geq 0$. From Step 3, (3.2), and Lemma 2.1(i), we have

$$
\begin{aligned}
\left\|v_{n}-x^{*}\right\|^{2} & =\left\|y_{n}-x^{*}\right\|^{2}-2 \alpha \eta_{n}\left\langle y_{n}-x^{*}, d_{n}\right\rangle+\left\|\alpha \eta_{n} d_{n}\right\|^{2} \\
& =\left\|y_{n}-x^{*}\right\|^{2}-2 \alpha \eta_{n}\left\langle y_{n}-w_{n}, d_{n}\right\rangle-2 \alpha \eta_{n}\left\langle w_{n}-x^{*}, d_{n}\right\rangle+\left\|\alpha \eta_{n} d_{n}\right\|^{2} \\
& \leq\left\|y_{n}-x^{*}\right\|^{2}-2 \alpha \eta_{n}\left\langle y_{n}-w_{n}, d_{n}\right\rangle+\left\|\alpha \eta_{n} d_{n}\right\|^{2} \\
& =\left\|y_{n}-x^{*}\right\|^{2}-\frac{2-\alpha}{\alpha}\left\|y_{n}-v_{n}\right\|^{2} .
\end{aligned}
$$

On the other hand, we have

$$
\left\|y_{n}-x^{*}\right\| \leq\left\|x_{n}-x^{*}\right\|+b_{n} \cdot \frac{\theta_{n}}{b_{n}}\left\|x_{n}-x_{n-1}\right\| .
$$

From Step 1, we have $\frac{\theta_{n}}{b_{n}}|| x_{n}-x_{n-1} \| \leq \frac{c_{n}}{b_{n}}$, which together with (A6) implies that $\lim _{n \rightarrow \infty} \frac{\theta_{n}}{b_{n}} \| x_{n}-$ $x_{n-1} \|=0$, so $\left\{\frac{\theta_{n}}{b_{n}}\left\|x_{n}-x_{n-1}\right\|\right\}$ is bounded. Thus, there exists a constant $M>0$ such that $\frac{\theta_{n}}{b_{n}}|| x_{n}-x_{n-1} \| \leq M$ for all $n \geq 1$. Hence, $\left\|y_{n}-x^{*}\right\| \leq\left\|x_{n}-x^{*}\right\|+b_{n} M$, which together with (3.3), and the fact that $\alpha \in(0,2)$, obtains

$$
\left\|v_{n}-x^{*}\right\| \leq\left\|y_{n}-x^{*}\right\| \leq\left\|x_{n}-x^{*}\right\|+b_{n} M .
$$

Furthermore, from Step 4 , the fact that $S$ is $\kappa$-generalized demimetric with $\kappa>0$, and $\lambda_{n}<$ $\frac{2}{\kappa|| B \|^{2}}$, we have

$$
\begin{aligned}
\left\|u_{n}-x^{*}\right\|^{2} & =\left\|v_{n}-x^{*}\right\|^{2}-2 \lambda_{n}\left\langle B v_{n}-B x^{*},(I-S) B v_{n}\right\rangle+\lambda_{n}^{2}\left\|B^{*}(I-S) B v_{n}\right\|^{2} \\
& \leq\left\|v_{n}-x^{*}\right\|^{2}-\frac{2 \lambda_{n}}{\kappa}\left\|(I-S) B v_{n}\right\|^{2}+\lambda_{n}^{2}\left\|B^{*}(I-S) B v_{n}\right\|^{2} \\
& \leq\left\|v_{n}-x^{*}\right\|^{2}+\lambda_{n}\left(\lambda_{n}\|B\|^{2}-\frac{2}{\kappa}\right)\left\|(I-S) B v_{n}\right\|^{2} \\
& \leq\left\|v_{n}-x^{*}\right\|^{2}
\end{aligned}
$$

From Lemma 2.2, (3.4) and (3.6), we have

$$
\begin{aligned}
\left\|x_{n+1}-x^{*}\right\| \leq & \left\|\left[\left(1-a_{n}\right) u_{n}-b_{n} \sigma F\left(u_{n}\right)\right]-\left[\left(1-a_{n}\right) x^{*}-b_{n} \sigma F\left(x^{*}\right)\right]\right\| \\
& +a_{n}\left\|x_{n}-x^{*}\right\|+b_{n} \sigma\left\|F\left(x^{*}\right)\right\| \\
\leq & \left(1-a_{n}-b_{n} \delta\right)\left\|u_{n}-x^{*}\right\|+a_{n}\left\|x_{n}-x^{*}\right\|+b_{n} \sigma\left\|F\left(x^{*}\right)\right\| \\
\leq & \left(1-a_{n}-b_{n} \delta\right)\left[\left\|x_{n}-x^{*}\right\|+b_{n} M\right]+a_{n}\left\|x_{n}-x^{*}\right\|+b_{n} \sigma\left\|F\left(x^{*}\right)\right\| \\
\leq & \left(1-b_{n} \delta\right)\left\|x_{n}-x^{*}\right\|+b_{n} \delta \frac{M+\sigma\left\|F\left(x^{*}\right)\right\|}{\delta} \\
\leq & \max \left\{\left\|x_{n}-x^{*}\right\|, \delta^{-1}\left(M+\sigma\left\|F\left(x^{*}\right)\right\|\right)\right\} .
\end{aligned}
$$


Hence,

$$
\left\|x_{n}-x^{*}\right\| \leq \max \left\{\left\|x_{1}-x^{*}\right\|, \delta^{-1}\left(M+\sigma\left\|F\left(x^{*}\right)\right\|\right)\right\} .
$$

This proves that $\left\{x_{n}\right\}$ is bounded, so are $\left\{u_{n}\right\},\left\{v_{n}\right\},\left\{y_{n}\right\}$, and $\left\{F u_{n}\right\}$.

Lemma 3.3. Let $\left\{y_{n}\right\}$ and $\left\{w_{n}\right\}$ be sequences generated by Algorithm 3.1 under Assumption 3.1. If there exist subsequences $\left\{y_{n_{k}}\right\}$ and $\left\{w_{n_{k}}\right\}$ of $\left\{y_{n}\right\}$ and $\left\{w_{n}\right\}$, respectively such that $\left\{y_{n_{k}}\right\}$ converges weakly to a point, say $z$ in $H_{1}$ and $\lim _{k \rightarrow \infty}\left\|w_{n_{k}}-y_{n_{k}}\right\|=0$, then $z \in \operatorname{VI}(C, A)$.

Proof. Since $w_{n_{k}}=P_{C}\left(y_{n_{k}}-\tau_{n_{k}} A y_{n_{k}}\right)$, we have $\left\langle y_{n_{k}}-\tau_{n_{k}} A y_{n_{k}}-w_{n_{k}}, x-w_{n_{k}}\right\rangle \leq 0, \forall x \in C$, which implies that

$$
\begin{aligned}
\left\langle y_{n_{k}}-w_{n_{k}}, x-w_{n_{k}}\right\rangle & \leq \tau_{n_{k}}\left\langle A y_{n_{k}}, x-w_{n_{k}}\right\rangle \\
& =\tau_{n_{k}}\left\langle A y_{n_{k}}, y_{n_{k}}-w_{n_{k}}\right\rangle+\tau_{n_{k}}\left\langle A y_{n_{k}}, x-y_{n_{k}}\right\rangle .
\end{aligned}
$$

Hence,

$$
\frac{1}{\tau_{n_{k}}}\left\langle y_{n_{k}}-w_{n_{k}}, x-w_{n_{k}}\right\rangle+\left\langle A y_{n_{k}}, w_{n_{k}}-y_{n_{k}}\right\rangle \leq\left\langle A y_{n_{k}}, x-y_{n_{k}}\right\rangle .
$$

Since $\left\{y_{n_{k}}\right\}$ converges weakly to a point $z \in H$, then it is bounded. Using the hypothesis $\| w_{n_{k}}-$ $y_{n_{k}} \| \rightarrow 0$ as $k \rightarrow \infty$, we have that $\left\{w_{n_{k}}\right\}$ and $\left\{A y_{n_{k}}\right\}$ are also bounded. From Lemma 3.1, we have $\lim _{k \rightarrow \infty} \tau_{n_{k}}>0$. Thus, passing limit as $k \rightarrow \infty$ in (3.7), we obtain $\liminf _{k \rightarrow \infty}\left\langle A y_{n_{k}}, x-y_{n_{k}}\right\rangle \geq 0$, $\forall x \in C$. We also have

$$
\begin{aligned}
\left\langle A w_{n_{k}}, x-w_{n_{k}}\right\rangle= & \left\langle A w_{n_{k}}-A y_{n_{k}}, x-y_{n_{k}}\right\rangle+\left\langle A y_{n_{k}}, x-y_{n_{k}}\right\rangle \\
& +\left\langle A w_{n_{k}}, y_{n_{k}}-w_{n_{k}}\right\rangle .
\end{aligned}
$$

From $\lim _{k \rightarrow \infty}|| y_{n_{k}}-w_{n_{k}} \|=0$, and the fact that $A$ is Lipschitz continuous, we have $\lim _{k \rightarrow \infty} \| A y_{n_{k}}-$ $A w_{n_{k}} \|=0$, which together with (3.8) yields that

$$
\liminf _{k \rightarrow \infty}\left\langle A w_{n_{k}}, x-w_{n_{k}}\right\rangle \geq 0, \forall x \in C .
$$

Now, let $\left\{\varepsilon_{k}\right\}$ be a decreasing sequence in $(0,1)$ such that $\varepsilon_{k} \rightarrow 0$ as $k \rightarrow \infty$. For each $k \geq 1$, we denote by $N_{k}$ the smallest nonnegative integer such that

$$
\left\langle A w_{n_{j}}, x-w_{n_{j}}\right\rangle+\varepsilon_{k} \geq 0, \forall j \geq N_{k} .
$$

It is clear that $\left\{N_{k}\right\}$ is increasing from the fact that $\left\{\varepsilon_{k}\right\}$ is decreasing. Also, for each $k \geq 1$, since $\left\{w_{N_{k}}\right\} \subset C$, we have $A\left(w_{N_{k}}\right) \neq 0$. Letting $z_{N_{k}}=\frac{A\left(w_{N_{k}}\right)}{\left\|A\left(w_{N_{k}}\right)\right\|^{2}}$, we obtain $\left\langle A\left(w_{N_{k}}\right), z_{N_{k}}\right\rangle=1$ for each $k \geq 1$. Thus, for each $k \geq 1,\left\langle A w_{N_{k}}, x+\varepsilon_{k} z_{N_{k}}-w_{N_{k}}\right\rangle \geq 0$. Since $A$ is pseudomonotone on $H_{1}$, we have $\left\langle A\left(x+\varepsilon_{k} z_{N_{k}}\right), x+\varepsilon_{k} z_{N_{k}}-w_{N_{k}}\right\rangle \geq 0$. Hence,

$$
\left\langle A x, x-w_{N_{k}}\right\rangle \geq\left\langle A x-A\left(x+\varepsilon_{k} z_{N_{k}}\right), x+\varepsilon_{k} z_{N_{k}}-w_{N_{k}}\right\rangle-\varepsilon_{k}\left\langle A x, z_{N_{k}}\right\rangle .
$$

Now, since $\left\{y_{n_{k}}\right\}$ converges weakly to $z$ and $\left\|w_{n_{k}}-y_{n_{k}}\right\| \rightarrow 0$ as $k \rightarrow \infty$, then we have that $\left\{w_{n_{k}}\right\}$ converges weakly to $z$ as $k \rightarrow \infty$. Since $\left\{w_{n_{k}}\right\} \subset C$, and $C$ is closed, then $z \in C$. We assume that $A z \neq 0$ (otherwise $z$ is a solution). From (A3), we obtain that $0<\|A z\| \leq \liminf _{k \rightarrow \infty}|| A w_{N_{k}} \|$. Also, 
using the fact that $\left\{w_{N_{k}}\right\} \subset\left\{w_{n_{k}}\right\}$ and $\varepsilon_{k} \rightarrow 0$ as $k \rightarrow \infty$, we have

$$
0 \leq \limsup _{k \rightarrow \infty}\left\|\varepsilon_{k} z_{N_{k}}\right\|=\limsup _{k \rightarrow \infty}\left(\frac{\varepsilon_{k}}{\left\|A w_{n_{k}}\right\|}\right) \leq \frac{\limsup _{k \rightarrow \infty} \varepsilon_{k}}{\liminf _{k \rightarrow \infty}|| A w_{n_{k}} \|}=0 .
$$

Hence, $\lim _{k \rightarrow \infty} \varepsilon_{k} z_{N_{k}}=0$. Thus, the right-hand side of (3.10) tends to zero as $k \rightarrow \infty$. That is, $\liminf _{k \rightarrow \infty}\left\langle A x, x-w_{N_{k}}\right\rangle \geq 0$. Therefore, for all $x \in C$,

$$
\langle A x, x-z\rangle=\lim _{k \rightarrow \infty}\left\langle A x, x-w_{N_{k}}\right\rangle=\liminf _{k \rightarrow \infty}\left\langle A x, x-w_{N_{k}}\right\rangle \geq 0
$$

From Lemma 2.3, we have that $z \in V I(C, A)$. This completes the proof.

Lemma 3.4. Let $\left\{x_{n}\right\}$ be a sequence generated by Algorithm 3.1 under Assumption 3.1. Then for all $n \geq 1$, we have

$$
\left.\left\|x_{n+1}-x^{*}\right\|^{2} \leq\left(1-b_{n} \delta\right)\left\|x_{n}-x^{*}\right\|^{2}+b_{n} \delta\left(\frac{\theta_{n}}{\delta b_{n}}\left\|x_{n}-x_{n-1}\right\| M_{1}+2 \frac{\sigma}{\delta}\left\langle F\left(x^{*}\right), x^{*}-x_{n+1}\right\rangle\right)\right\}_{3} .
$$

where $x^{*}$ is the unique solution of the BSVIP (1.12).

Proof. From Lemma 2.1 and Lemma 2.2, we have

$$
\begin{aligned}
\left\|x_{n+1}-x^{*}\right\|^{2}= & \|\left\{\left[\left(1-a_{n}\right) u_{n}-b_{n} \sigma F\left(u_{n}\right)\right]-\left[\left(1-a_{n}\right) x^{*}-b_{n} \sigma F\left(x^{*}\right)\right]\right\} \\
& +a_{n}\left(x_{n}-x^{*}\right)-b_{n} \sigma F\left(x^{*}\right) \|^{2} \\
\leq & \left\|\left[\left(1-a_{n}\right) u_{n}-b_{n} \sigma F\left(u_{n}\right)\right]-\left[\left(1-a_{n}\right) x^{*}-b_{n} \sigma F\left(x^{*}\right)\right]+a_{n}\left(x_{n}-x^{*}\right)\right\|^{2} \\
& +2 b_{n} \sigma\left\langle F\left(x^{*}\right), x^{*}-x_{n+1}\right\rangle \\
\leq & \left\{\left\|\left[\left(1-a_{n}\right) u_{n}-b_{n} \sigma F\left(u_{n}\right)\right]-\left[\left(1-a_{n}\right) x^{*}-b_{n} \sigma F\left(x^{*}\right)\right]\right\|+a_{n}\left\|x_{n}-x^{*}\right\|\right\}^{2} \\
& +2 b_{n} \sigma\left\langle F\left(x^{*}\right), x^{*}-x_{n+1}\right\rangle \\
\leq & {\left[1-a_{n}-b_{n} \delta\right]\left\|u_{n}-x^{*}\right\|^{2}+a_{n}\left\|x_{n}-x^{*}\right\|^{2}+2 b_{n} \sigma\left\langle F\left(x^{*}\right), x^{*}-x_{n+1}\right\rangle . }
\end{aligned}
$$

From Step 2, we have

$$
\begin{aligned}
\left\|y_{n}-x^{*}\right\|^{2} & =\left\|x_{n}-x^{*}\right\|^{2}+2 \theta_{n}\left\langle x_{n}-x^{*}, x_{n}-x_{n-1}\right\rangle+\theta_{n}^{2}\left\|x_{n}-x_{n-1}\right\|^{2} \\
& \leq\left\|x_{n}-x^{*}\right\|^{2}+\theta_{n}\left\|x_{n}-x^{*}\right\|\left[2|| x_{n}-x_{n-1}\left\|+\theta_{n}\right\| x_{n}-x_{n-1} \|\right] \\
& \leq\left\|x_{n}-x^{*}\right\|^{2}+\theta_{n}\left\|x_{n}-x_{n-1}\right\| M_{1}
\end{aligned}
$$

for some constant $M_{1}>0$. So, combining (3.3), (3.5), and (3.12), we obtain

$$
\begin{aligned}
\left\|u_{n}-x^{*}\right\|^{2} \leq & \left\|x_{n}-x^{*}\right\|^{2}+\theta_{n}\left\|x_{n}-x_{n-1}\right\| M_{1}-\frac{2-\alpha}{\alpha}\left\|y_{n}-v_{n}\right\|^{2} \\
& +\lambda_{n}\left(\lambda_{n}\|B\|^{2}-\frac{2}{\kappa}\right)\left\|(I-S) B v_{n}\right\|^{2} .
\end{aligned}
$$


From (3.12) and (3.13), we have

$$
\begin{aligned}
\left\|x_{n+1}-x^{*}\right\|^{2} \leq & \left(1-a_{n}-b_{n} \delta\right)\left\|x_{n}-x^{*}\right\|^{2}+\theta_{n}\left(1-a_{n}-b_{n} \delta\right)\left\|x_{n}-x_{n-1}\right\| M_{1} \\
& -\frac{2-\alpha}{\alpha}\left(1-a_{n}-b_{n} \delta\right)\left\|y_{n}-v_{n}\right\|^{2} \\
& +\lambda_{n}\left(1-a_{n}-b_{n} \delta\right)\left(\lambda_{n}\|B\|^{2}-\frac{2}{\kappa}\right)\left\|(I-S) B v_{n}\right\|^{2} \\
& +a_{n}\left\|x_{n}-x^{*}\right\|^{2}+2 b_{n} \sigma\left\langle F\left(x^{*}\right), x^{*}-x_{n+1}\right\rangle \\
\leq & \left(1-b_{n} \delta\right)\left\|x_{n}-x^{*}\right\|^{2}+\theta_{n}\left\|x_{n}-x_{n-1}\right\| M_{1}-\frac{2-\alpha}{\alpha}\left(1-a_{n}-b_{n} \delta\right)\left\|y_{n}-v_{n}\right\|^{2} \\
& +\lambda_{n}\left(1-a_{n}-b_{n} \delta\right)\left(\lambda_{n}\|B\|^{2}-\frac{2}{\kappa}\right)\left\|(I-S) B v_{n}\right\|^{2}+2 b_{n} \sigma\left\langle F\left(x^{*}\right), x^{*}-x_{n+1}\right\rangle \\
\leq & \left(1-b_{n} \delta\right)\left\|x_{n}-x^{*}\right\|^{2}+\theta_{n}\left\|x_{n}-x_{n-1}\right\| M_{1}+2 b_{n} \sigma\left\langle F\left(x^{*}\right), x^{*}-x_{n+1}\right\rangle,
\end{aligned}
$$

which yields the desired conclusion.

Theorem 3.1. Let $\left\{x_{n}\right\}$ be a sequence generated by Algorithm 3.1 under Assumption 3.1. Then, $\left\{x_{n}\right\}$ converges strongly to the unique solution of the BSVIP (1.12).

Proof. From Lemma 2.4 and (3.11), we only need to show that

$$
\limsup _{k \rightarrow \infty}\left(\frac{\theta_{n_{k}}}{\delta b_{n_{k}}}\left\|x_{n_{k}}-x_{n_{k}-1}\right\|+2 \frac{\sigma}{\delta}\left\langle F\left(x^{*}\right), x^{*}-x_{n_{k}+1}\right\rangle\right) \leq 0
$$

for every subsequence $\left\{\| x_{n_{k}}-x^{*}||\right\}$ of $\left\{|| x_{n}-x^{*} \|\right\}$ satisfying the following condition:

$$
\liminf _{k \rightarrow \infty}\left(\left\|x_{n_{k}+1}-x^{*}\right\|-\left\|x_{n_{k}}-x^{*}\right\|\right) \geq 0 \text {. }
$$

Now, let $\left\{\left\|x_{n_{k}}-x^{*}\right\|\right\}$ be a subsequence of $\left\{\left\|x_{n}-x^{*}\right\|\right\}$ such that (3.14) holds. Then

$$
\begin{aligned}
& \liminf _{k \rightarrow \infty}\left(\left\|x_{n_{k}+1}-x^{*}\right\|^{2}-\left\|x_{n_{k}}-x^{*}\right\|^{2}\right) \\
& \quad=\liminf _{k \rightarrow \infty}\left[\left(\left\|x_{n_{k}+1}-x^{*}\right\|-\left\|x_{n_{k}}-x^{*}\right\|\right)\left(\left\|x_{n_{k}+1}-x^{*}\right\|+\left\|x_{n_{k}}-x^{*}\right\|\right)\right] \\
& \geq 0 .
\end{aligned}
$$

Thus, from (3.14) and (3.15), we obtain

$$
\begin{aligned}
& \limsup _{k \rightarrow \infty}\left(\frac{2-\alpha}{\alpha}\left(1-a_{n_{k}}-\delta b_{n_{k}}\right)\left\|y_{n_{k}}-v_{n_{k}}\right\|^{2}\right. \\
& \left.+\lambda_{n_{k}}\left(\frac{2}{\kappa}-\lambda_{n_{k}}\|B\|^{2}\right)\left(1-a_{n_{k}}-\delta b_{n_{k}}\right)\left\|(I-S) B v_{n_{k}}\right\|^{2}\right) \\
\leq & \limsup _{k \rightarrow \infty}\left(\left(1-b_{n_{k}} \delta\right)\left\|x_{n_{k}}-x^{*}\right\|^{2}+b_{n_{k}} \cdot \frac{\theta_{n_{k}}}{b_{n_{k}}}\left\|x_{n_{k}}-x_{n_{k}-1}\right\| M_{1}\right. \\
& \left.+2 b_{n_{k}} \sigma\left\|F\left(x^{*}\right)\right\|\left\|x_{n_{k}+1}-x^{*}\right\|-\left\|x_{n_{k}+1}-x^{*}\right\|^{2}\right) \\
= & -\liminf _{k \rightarrow \infty}\left(\left\|x_{n_{k}+1}-x^{*}\right\|^{2}-\left\|x_{n_{k}}-x^{*}\right\|^{2}\right) \leq 0,
\end{aligned}
$$

which implies that

$$
\lim _{k \rightarrow \infty}\left(\frac{2-\alpha}{\alpha}\left(1-a_{n_{k}}-\delta b_{n_{k}}\right)\left\|y_{n_{k}}-v_{n_{k}}\right\|^{2}+\lambda_{n_{k}}\left(\frac{2}{\kappa}-\lambda_{n_{k}}\|B\|^{2}\right)\left(1-a_{n_{k}}-\delta b_{n_{k}}\right)\left\|(I-S) B v_{n_{k}}\right\|^{2}\right)=0 .
$$


Thus,

$$
\lim _{k \rightarrow \infty}\left\|y_{n_{k}}-v_{n_{k}}\right\|=0
$$

and

$$
\lim _{k \rightarrow \infty}\left\|(I-S) B v_{n_{k}}\right\|=0
$$

On the other hand, we have

$$
\begin{aligned}
\left\|d_{n_{k}}\right\| & \leq\left\|y_{n_{k}}-w_{n_{k}}\right\|+\tau_{n_{k}}|| A y_{n_{k}}-A w_{n_{k}} \| \\
& \leq\left(1+\mu \frac{\tau_{n_{k}}}{\tau_{n_{k}+1}}\right)\left\|y_{n_{k}}-w_{n_{k}}\right\|
\end{aligned}
$$

and

$$
\begin{aligned}
\left\langle y_{n_{k}}-w_{n_{k}}, d_{n_{k}}\right\rangle & =\left\|y_{n_{k}}-w_{n_{k}}\right\|^{2}-\tau_{n_{k}}\left\langle y_{n_{k}}-w_{n_{k}}, A y_{n_{k}}-A w_{n_{k}}\right\rangle \\
& \geq\left\|y_{n_{k}}-w_{n_{k}}\right\|^{2}-\tau_{n_{k}}|| y_{n_{k}}-w_{n_{k}}|| \mid A y_{n_{k}}-A w_{n_{k}} \| \\
& \geq\left(1-\mu \frac{\tau_{n_{k}}}{\tau_{n_{k}+1}}\right)\left\|y_{n_{k}}-w_{n_{k}}\right\|^{2} .
\end{aligned}
$$

Since $v_{n_{k}}=y_{n_{k}}-\alpha \eta_{n_{k}} d_{n_{k}}$, we find from (3.18) and (3.19) that

$$
\left\|v_{n_{k}}-y_{n_{k}}\right\|=\alpha \eta_{n_{k}}|| d_{n_{k}}\left\|=\frac{\alpha \cdot\left\langle y_{n_{k}}-w_{n_{k}}, d_{n_{k}}\right\rangle}{\left\|d_{n_{k}}\right\|} \geq \frac{\alpha\left(\tau_{n_{k}+1}-\mu \tau_{n_{k}}\right)}{\tau_{n_{k}+1}+\mu \tau_{n_{k}}}\right\| y_{n_{k}}-w_{n_{k}} \|,
$$

which implies that

$$
\left\|y_{n_{k}}-w_{n_{k}}\right\| \leq \frac{\tau_{n_{k}+1}+\mu \tau_{n_{k}}}{\alpha\left(\tau_{n_{k}+1}-\mu \tau_{n_{k}}\right)}\left\|v_{n_{k}}-y_{n_{k}}\right\| .
$$

From Lemma 3.1, we have

$$
\lim _{k \rightarrow \infty} \frac{\tau_{n_{k}+1}+\mu \tau_{n_{k}}}{\tau_{n_{k}+1}-\mu \tau_{n_{k}}}=\frac{1+\mu}{1-\mu}
$$

which shows that $\left\{\frac{\tau_{n_{k}+1}+\mu \tau_{n_{k}}}{\tau_{n_{k}+1}-\mu \tau_{n_{k}}}\right\}$ is bounded. Hence, combining (3.16) and (3.20) yields that $\lim _{k \rightarrow \infty}\left\|y_{n_{k}}-w_{n_{k}}\right\|=0$. This together with (3.16) implies $\lim _{k \rightarrow \infty}\left\|v_{n_{k}}-w_{n_{k}}\right\|=0$. Observe that

$$
\left\|y_{n_{k}}-x_{n_{k}}\right\|=b_{n_{k}} \cdot \frac{\theta_{n_{k}}}{b_{n_{k}}}\left\|x_{n_{k}}-x_{n_{k}-1}\right\| \rightarrow 0 \text { as } k \rightarrow \infty .
$$

Hence, we have $\lim _{k \rightarrow \infty}\left\|x_{n_{k}}-v_{n_{k}}\right\|=0$, and $\lim _{k \rightarrow \infty}\left\|x_{n_{k}}-w_{n_{k}}\right\|=0$. Since $\left\{x_{n_{k}}\right\}$ is bounded, there exists a subsequence say $\left\{x_{n_{k_{j}}}\right\}$ of $\left\{x_{n_{k}}\right\}$ that converges weakly to say $z \in H_{1}$ as $j \rightarrow \infty$. Then, from (3.21), we have that $\left\{y_{n_{k_{j}}}\right\}$ converges weakly to $z$ as $j \rightarrow \infty$. From Lemma 3.3, we obtain that $z \in V I(C, A)$.

Next, we show that $B z \in F(S)$. Observe that $\left\{v_{n_{k_{j}}}\right\}$ converges weakly to $z$ as $j \rightarrow \infty$, and $B$ is a bounded linear operator. Then, $\left\{B v_{n_{k_{j}}}\right\}$ converges weakly to $B z \in H_{2}$. Combining (3.17) and demiclosedness of $S$, we obtain that $B z \in F(S)$. In view of Step 4 and (3.17), we have that $\left\|x_{n_{k}}-u_{n_{k}}\right\| \leq\left\|x_{n_{k}}-v_{n_{k}}\right\|+\lambda_{n_{k}}|| B^{*}||\left\|(I-S) B v_{n_{k}}\right\| \rightarrow 0$ as $k \rightarrow \infty$. Thus

$$
\left\|x_{n_{k}+1}-x_{n_{k}}\right\| \leq\left(1-a_{n_{k}}\right)\left\|u_{n_{k}}-x_{n_{k}}\right\|+b_{n_{k}} \sigma\left\|F\left(u_{n_{k}}\right)\right\| \rightarrow 0 \text { as } k \rightarrow \infty \text {. }
$$


Since $x_{n_{k_{j}}} \rightarrow z$, we have $\limsup _{k \rightarrow \infty}\left\langle F\left(x^{*}\right), x^{*}-x_{n_{k}}\right\rangle=\lim _{j \rightarrow \infty}\left\langle F\left(x^{*}\right), x^{*}-x_{n_{k_{j}}}\right\rangle=\left\langle F\left(x^{*}\right), x^{*}-z\right\rangle$. Since $x^{*}$ is the unique solution of BSVIP in (1.12), we have

$$
\limsup _{k \rightarrow \infty}\left\langle F\left(x^{*}\right), x^{*}-x_{n_{k}}\right\rangle=\left\langle F\left(x^{*}\right), x^{*}-z\right\rangle \leq 0 .
$$

Thus, from (3.22) and (3.23), we have

$$
\limsup _{k \rightarrow \infty}\left\langle F\left(x^{*}\right), x^{*}-x_{n_{k}+1}\right\rangle=\limsup _{k \rightarrow \infty}\left\langle F\left(x^{*}\right), x^{*}-x_{n_{k}}\right\rangle=\left\langle F\left(x^{*}\right), x^{*}-z\right\rangle \leq 0 .
$$

In view of Lemma 2.4, we conclude that $\lim _{n \rightarrow \infty}|| x_{n}-x^{*} \|=0$. Therefore, $\left\{x_{n}\right\}$ converges strongly to $x^{*}$ as $n \rightarrow \infty$.

\section{NUMERICAL EXPERIMENTS}

In this section, we discuss the numerical behavior of Algorithm 3.1 in both finite and infinite dimensional Hilbert spaces. We also compare our method with the methods of Thong et al. [32] (Algorithm (1.9)) and Tan et al. [33] (Algorithm (1.10) and Algorithm (1.11)). All codes are written in Matlab 2016 (b) and performed on a personal computer with an Intel(R) Core(TM) i5$2600 \mathrm{CPU}$ at $2.30 \mathrm{GHz}$ and $8.00 \mathrm{~Gb}-\mathrm{RAM}$. In Tables 1-2, "Iter." means the number of iterations while "CPU" means the CPU time in seconds. In our computations, we choose $\tau_{1}=1, \alpha=$ $1.1, \mu=0.5$ and $\sigma=\frac{\beta}{L^{2}}$. Also, we take $b_{n}=\frac{1}{n+1}, a_{n}=\frac{1}{2}-b_{n}, \lambda_{n}=\frac{1}{\kappa|\|B\||^{2}}$, and $\theta_{n}=\bar{\theta}_{n}$ with $c_{n}=\frac{b_{n}}{n^{0.01}}$ and $\theta=0.3$.

Example 4.1. Let $H_{1}=\mathbb{R}^{N}$ and $H_{2}=\mathbb{R}^{m}$. Define $A: \mathbb{R}^{N} \rightarrow \mathbb{R}^{N}$ by $A(x)=M x+q$, where the matrix $M$ is formed as: $M=V \sum V^{\prime}$, where $V=I-\frac{2 v v^{\prime}}{\|v\|^{2}}$ and $\sum=\operatorname{diag}\left(\sigma_{11}, \sigma_{12}, \cdots, \sigma_{1 N}\right)$ are the Householder and the diagonal matrix, and

$$
\sigma_{1 j}=\cos \frac{j \pi}{N+1}+1+\frac{\cos \frac{\pi}{N+1}+1-\widehat{C}\left(\cos \frac{N \pi}{N+1}+1\right)}{\widehat{C}-1}, j=1,2, \cdots, N
$$

with $\widehat{C}$ being the present condition number of $M$ ([40, Example 5.2]). In the numerical computation, we choose $\widehat{C}=10^{4}, q=0$, and uniformly take the vector $v \in \mathbb{R}^{N}$ in $(-1,1)$. Thus, $A$ is pseudomonotone and Lipschitz continuous with $K=\|M\|$ (see [40]). Furthermore, we generate the bounded linear operator $B \in \mathbb{R}^{M \times N}$ with independent Gaussian components distributed in the interval $(0,1)$, and then normalize each column of $B$ with the unit norm. We set $C=\left\{x \in \mathbb{R}^{N}:\|x\| \leq 1\right\}$. The projection onto $C$ is effectively computed in Matlab. We define $F: \mathbb{R}^{N} \rightarrow \mathbb{R}^{N}$ by $F x=D x+p$, where $D$ is a symmetric and positive-definite matrix of size $N \times N$ and $p$ is a vector in $\mathbb{R}^{N}$. Clearly, $F$ is $\|D\|$-Lipschitz continuous. Moreover, since $D$ is symmetric and positive-definite, we have that $\langle D x-D y\rangle=\langle D(x-y)\rangle \geq\|D\|^{-1}\|x-y\|^{2}$. Hence, $F$ is strongly monotone. Also, define $S: \mathbb{R}^{m} \rightarrow \mathbb{R}^{m}$ by $S x=2 x$. Since the solution of the problem is unknown, we define the sequence $\mathrm{TOL}_{n}:=|| x_{n+1}-x_{n} \|^{2}$, and use the stopping criterion $\mathrm{TOL}_{n}<\varepsilon$ for the iterative processes, where $\varepsilon$ is the predetermined error. Moreover, we consider different scenarios of the dimensions. That is, $N=100,300,500,1000$, and $m=N / 2$ with starting points $x_{1}=(1,1, \ldots, 1)^{\prime}$ and $x_{0}=(0,0, \ldots, 0)^{\prime}$. For this example, we take $\varepsilon=10^{-5}$ as the stopping criterion and obtain the numerical results reported in Table 1 and Figure 1. 

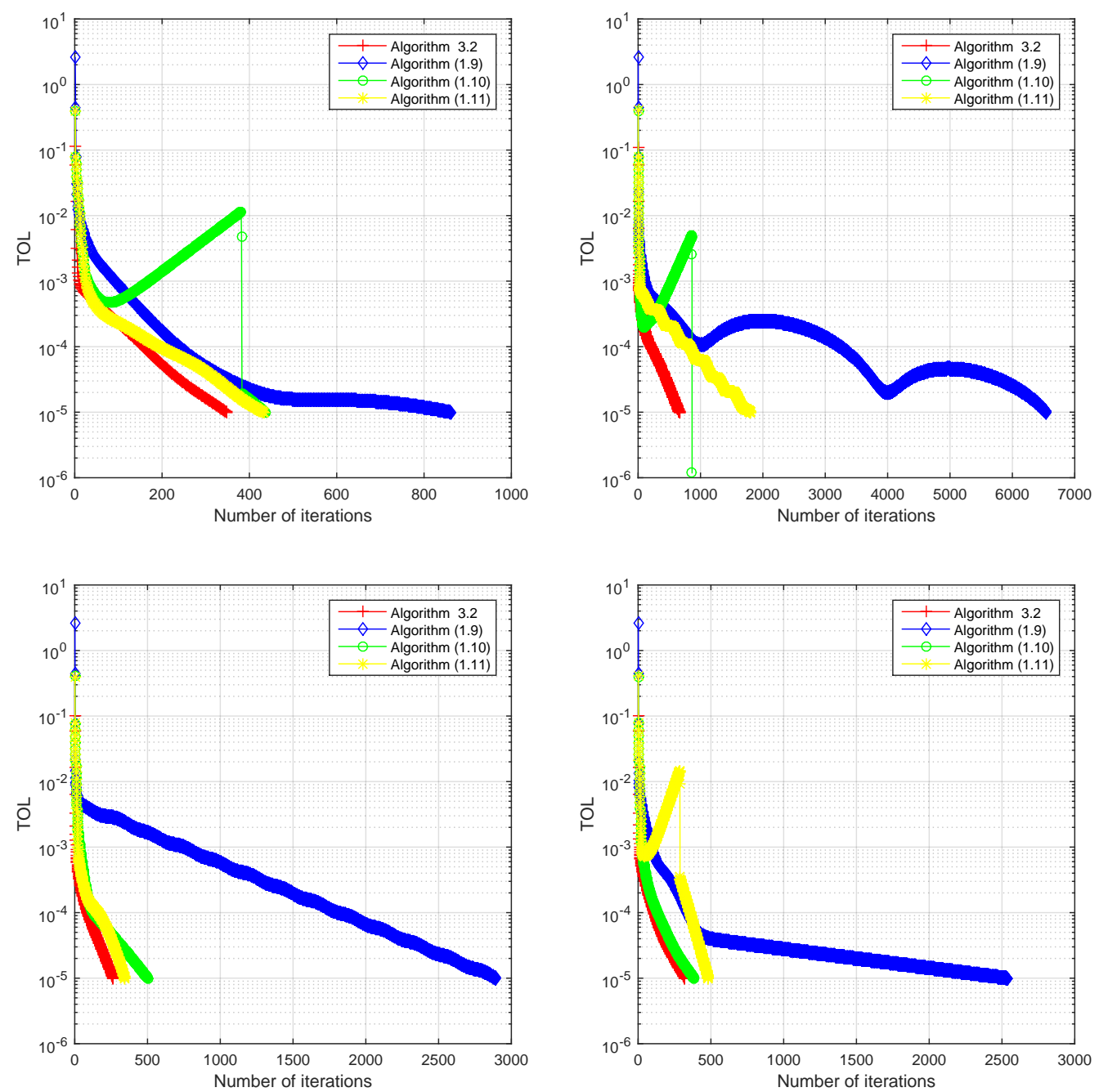

FIGURE 1. The behavior of TOL $_{n}$ with $\varepsilon=10^{-5}$ for Example 4.1: Top Left: $(N, m)=(100,50)$; Top Right: $(N, m)=(300,150)$; Bottom Left: $(N, m)=$ (500, 250); Bottom Right: $(N, m)=(1000,500)$.

Table 1. Numerical results for Example 4.1 with $\varepsilon=10^{-5}$.

\begin{tabular}{llllll}
\hline$(N, m)$ & & $\begin{array}{l}\text { Algorithm } \\
3.1\end{array}$ & $\begin{array}{l}\text { Algorithm } \\
(1.9)\end{array}$ & $\begin{array}{l}\text { Algorithm } \\
(1.10)\end{array}$ & $\begin{array}{l}\text { Algorithm } \\
(1.11)\end{array}$ \\
\hline$(100,50)$ & CPU Iter. & 0.0371 & 1.1456 & 0.4401 & 0.4236 \\
& & 348 & 862 & 437 & 433 \\
$(300,150)$ & CPU Iter. & 0.0385 & 1.2824 & 0.4426 & 0.5568 \\
& & 662 & 6548 & 865 & 1797 \\
$(500,250)$ & CPU Iter. & 0.0180 & 1.1178 & 0.4499 & 0.1124 \\
& & 265 & 2893 & 501 & 344 \\
$(1000,500)$ & CPU Iter. & 0.0259 & 1.1948 & 0.1185 & 0.1134 \\
& & 322 & 2540 & 384 & 483
\end{tabular}


Example 4.2. Let $H_{1}=\left(l_{2}(\mathbb{R}),\|\cdot\|_{l_{2}}\right)=H_{2}$, where $l_{2}(\mathbb{R}):=\left\{x=\left(x_{1}, x_{2}, x_{3}, \ldots\right), x_{i} \in \mathbb{R}\right.$ : $\left.\sum_{i=1}^{\infty}\left|x_{i}\right|^{2}<\infty\right\}$ and $\|x\|_{l_{2}}:=\left(\sum_{i=1}^{\infty}\left|x_{i}\right|^{2}\right)^{\frac{1}{2}}, \forall x \in l_{2}(\mathbb{R})$. Now, define the operator $B: l_{2}(\mathbb{R}) \rightarrow l_{2}(\mathbb{R})$ by $B x=\left(0, x_{1}, \frac{x_{2}}{2}, \frac{x_{3}}{3}, \ldots\right), \forall x \in l_{2}(\mathbb{R})$. Then, $B$ is a bounded linear operator on $l_{2}(\mathbb{R})$ with adjoint $B^{*} y=\left(y_{2}, \frac{y_{3}}{2}, \frac{y_{4}}{3}, \ldots\right), \forall y \in l_{2}(\mathbb{R})$. Let $C=\left\{x \in l_{2}(\mathbb{R}):\left|x_{i}\right| \leq \frac{1}{i}, i=1,2,3, \ldots\right\}$. Thus, we have explicit formula for $P_{C}$. Now, define the operator $A: l_{2}(\mathbb{R}) \rightarrow l_{2}(\mathbb{R})$ by

$$
A x=\left(\|x\|+\frac{1}{\|x\|+\alpha}\right) \alpha,
$$

for some $\alpha>0$. Then, $A$ is pseudomonotone on $l_{2}(\mathbb{R})$ (see [41]). Furthermore, define the mapping $S: l_{2}(\mathbb{R}) \rightarrow l_{2}(\mathbb{R})$ by $S x=\left(0, x_{1}, x_{2}, \ldots\right)$, and $F: l_{2}(\mathbb{R}) \rightarrow l_{2}(\mathbb{R})$ by $F x=x-x_{0}$. Then, $F$ is strongly monotone and Lipschitz continuous.

We take $\varepsilon=10^{-8}$ as the stopping criterion and choose the starting points as follows:

Case 1: Take $x_{1}=\left(1, \frac{1}{2}, \frac{1}{3}, \cdots\right)$ and $x_{0}=\left(\frac{1}{2}, \frac{1}{5}, \frac{1}{10}, \cdots\right)$.

Case 2: Take $x_{1}=\left(\frac{1}{2}, \frac{1}{5}, \frac{1}{10}, \cdots\right)$ and $x_{0}=\left(1, \frac{1}{2}, \frac{1}{3}, \cdots\right)$.

Case 3: Take $x_{1}=\left(1, \frac{1}{4}, \frac{1}{9}, \cdots\right)$ and $x_{0}=\left(\frac{1}{2}, \frac{1}{4}, \frac{1}{8}, \cdots\right)$.

Case 4: Take $x_{1}=\left(\frac{1}{2}, \frac{1}{4}, \frac{1}{8}, \cdots\right)$ and $x_{0}=\left(1, \frac{1}{4}, \frac{1}{9}, \cdots\right)$.

The numerical results reported in Table 2 and Figure 2.

Table 2. Numerical results for Example 4.2 with $\varepsilon=10^{-8}$.

\begin{tabular}{|c|c|c|c|c|c|}
\hline Cases & & $\begin{array}{l}\text { Algorithm } \\
3.1\end{array}$ & $\begin{array}{l}\text { Algorithm } \\
(1.9)\end{array}$ & $\begin{array}{l}\text { Algorithm } \\
(1.10)\end{array}$ & $\begin{array}{l}\text { Algorithm } \\
\text { (1.11) }\end{array}$ \\
\hline \multirow[t]{2}{*}{1} & CPU Iter. & 0.0654 & 1.0643 & 1.1005 & 1.0513 \\
\hline & & 330 & 667 & 679 & 579 \\
\hline \multirow[t]{2}{*}{2} & CPU Iter. & 0.0340 & 1.0520 & 1.0709 & 1.0311 \\
\hline & & 410 & 809 & 817 & 705 \\
\hline \multirow[t]{2}{*}{3} & CPU Iter. & 0.0349 & 1.0517 & 1.0998 & 1.0290 \\
\hline & & 292 & 587 & 609 & 506 \\
\hline \multirow[t]{2}{*}{4} & CPU Iter. & 0.0377 & 1.0722 & 1.0572 & 1.0298 \\
\hline & & 329 & 668 & 673 & 582 \\
\hline
\end{tabular}

\section{Conclusion}

A modified projection and contraction method with inertial extrapolation steps was introduced and studied for solving the bilevel split variational inequality problem in two real Hilbert spaces when one of the cost operators is pseudomonotone, Lipschitz continuous, but not sequentially weakly continuous. We proved that the proposed algorithm converges strongly to the unique solution of the bilevel split variational inequality problem. Finally, we considered some numerical examples of our proposed method in comparison with other state-of-the-art methods for solving the bilevel split variational inequality problem. In all our comparisons, the numerical results reveal that our method performs better than these other methods.

\section{Acknowledgements}

The authors sincerely thank the anonymous referees and the editor for their careful reading, constructive comments and fruitful suggestions that substantially improved the manuscript. 

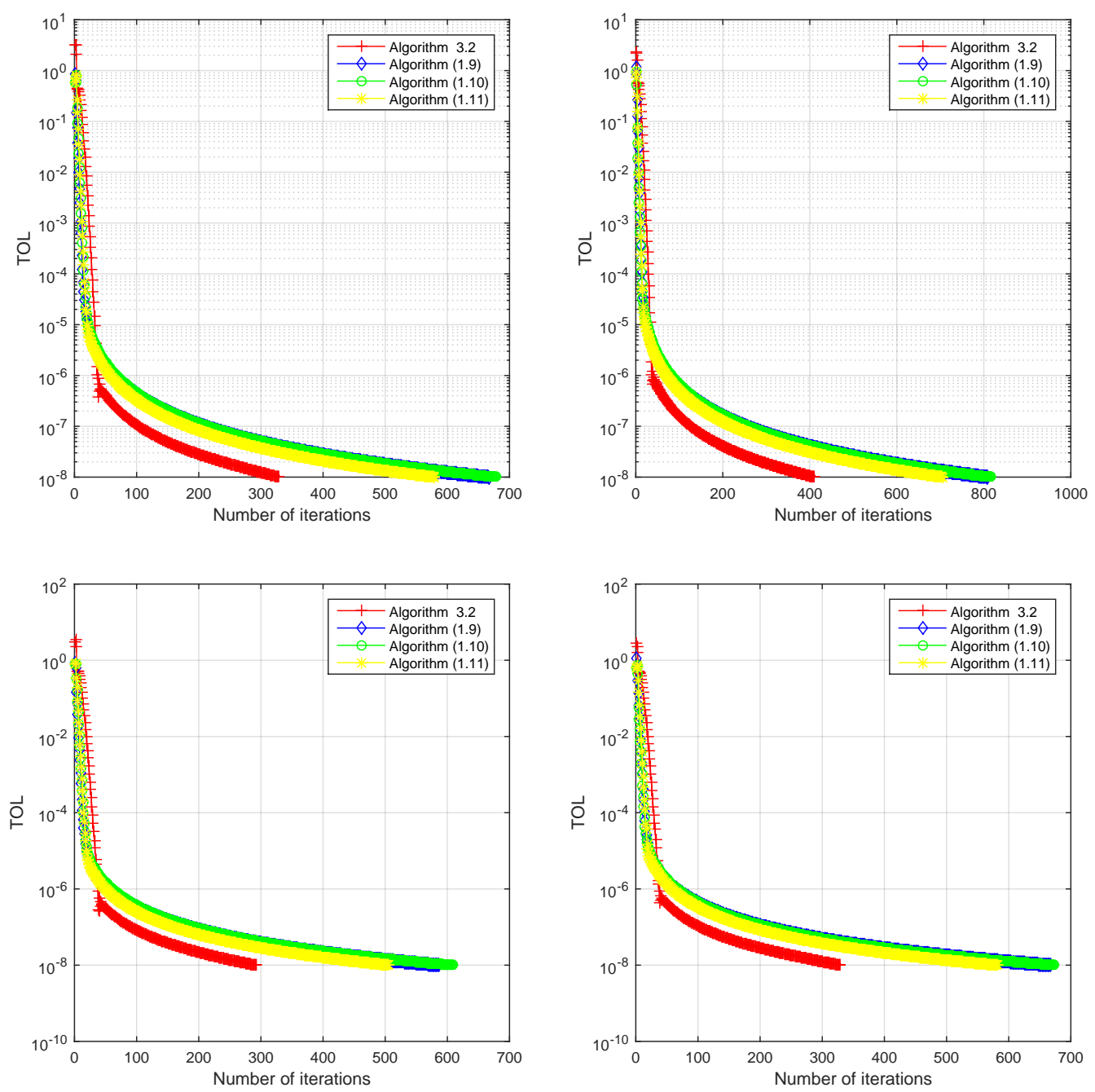

FIgurE 2. The behavior of $\mathrm{TOL}_{n}$ with $\varepsilon=10^{-8}$ for Example 4.2: Top Left: Case 1; Top Right: Case 2; Bottom Left: Case 3; Bottom Right: Case 4.

\section{REFERENCES}

[1] Y. Censor, A. Gibali, S. Reich, Algorithms for the split variational inequality problem, Numer. Algo. 59 (2012), 301-323.

[2] G. Stampacchi, Formes bilineaires coercivites sur les ensembles convexes, C. R. Acad. Sciences, Paris, 258 (1964), 4413-4416.

[3] S. C. Dafermos, Tracffic equilibrium and variational inequalities, Trans. Sci. 14 (1980), 1-13.

[4] S. C. Dafermos, F. T. Sparrow, The traffic assignment problem for a general network, J. Res. Nat. Bur. Standard 73B, (1969), 91-117.

[5] N.T. An, P.D. Dong, X. Qin, N.T. An, P.D. Dong, X. Qin, Robust feature selection via nonconvex sparsitybased methods, J. Nonlinear Var. Anal. 5 (2021), 59-77.

[6] Y. Censor, T. Elfving, A multiprojection algorithm using Bregman projections in product space, Numer. Algo. 8 (1994), 221-239.

[7] C. Byrne, A unified treatment for some iterative algorithms in signal processing and image reconstruction, Inverse Probl. 20 (2004), 103-120. 
[8] Y. Censor, T. Bortfeld, B. Marti, A. Trofimov, A unified approach for inversion problems in intensity modulated radiation therapy, Phys. Med. Biol. 51 (2006), 2353-2365.

[9] T. Humphries, M. Loreto, B. Halter, W. O'Keeffe, L. Ramirez, Comparison of regularized and superiorized methods for tomographic image reconstruction, J. Appl. Numer. Optim. 2 (2020), 77-99.

[10] G. M. Korpelevich, The extragradient method for finding saddle points and other problems, Ekonom Mate Metod 12 (1976), 747-756.

[11] A. S. Antipin, On a method for convex programs using a symmetrical modification of the Lagrange function, Ekonom I Mate Metod 6 (1976), 1164-1173.

[12] Y. Censor, A. Gibali, S. Reich, The subgradient extragradient method for solving variational inequalities in Hilbert space, J. Optim. Theory Appl. 148 (2011), 318-335.

[13] P. Tseng, A modified forward-backward splitting method for maximal monotone mappings, SIAM J. Control Optim. 38 (2000), 431-446.

[14] L.C. Ceng, A subgradient-extragradient method for bilevel equilibrium problems with the constraints of variational inclusion systems and fixed point problems, Commun. Optim. Theory, 2021 (2021), Article ID 4.

[15] L. Liu, B. Tan, S. Y. Cho, On the resolution of variational inequality problems with a double-hierarchical structure, J. Nonlinear Convex Anal. 21 (2020), 377-386.

[16] M. Tian, G. Xu, Inertial modified Tseng's extragradient algorithms for solving monotone variational inequalities and fixed point problems, J. Nonlinear Funct. Anal. 2020 (2020), Article ID 35.

[17] L.C. Ceng, A. Petruse, X. Qin, J.C. Yao, A modified inertial subgradient extragradient method for solving pseudomonotone variational inequalities and common fixed point problems, Fixed Point Theory 21 (2020), 93-108.

[18] B. S. He, A class of projection and contraction methods for monotone variational inequalities, Appl. Math. Optim. 35 (1997), 69-76.

[19] D. F. Sun, A class of iterative methods for solving nonlinear projection equations, J. Optim. Theory Appl. 91 (1996), 123-140.

[20] D. V. Thong, N.T. Vinh, Y.J. Cho, New strong convergence theorem of the inertial projection and contraction method for variational inequality problems, Numer. Algo. 84 (2020), 285-305.

[21] M. Tian, B.N. Jiang, Inertial hybrid algorithm for variational inequality problems in Hilbert spaces, J. Inequal. Appl. 2020 (2020), 12.

[22] B. Tan, X. Qin, J.C. Yao, Strong convergence of self-adaptive inertial algorithms for solving split variational inclusion problems with applications, J. Comput. Sci. 87 (2021), 20.

[23] B.T. Polyak, Some methods of speeding up the convergence of iteration methods, U.S.S.R. Comput. Math. Math. Phys. 4 (1964), 1-17.

[24] F. Alvarez, Weak convergence of a relaxed and inertial hybrid projection-proximal point algorithm for maximal monotone operators in Hilbert space, SIAM J. Optim. 14 (2004), 773-782.

[25] X. Qin, L. Wang, J.C. Yao, Inertial splitting method for maximal monotone mappings, J. Nonlinear Convex Anal. 21 (2020), 2325-2333.

[26] Y. Shehu, J.C. Yao, Rate of convergence for inertial iterative method for countable family of certain quasinonexpansive mappings, J. Nonlinear Convex Anal. 21 (2020), 533-541.

[27] B. Tan, S.Y. Cho, Strong convergence of inertial forward-backward methods for solving monotone inclusions, Appl. Anal. (2021), 10.1080/00036811.2021.1892080.

[28] P. Cholamjiak, D. V. Thong, Y.J. Cho, A novel inertial projection and contraction method for solving pseudomonotone variational inequality problem, Acta Appl. Math. 169 (2020), 217-245.

[29] Z. Q. Luo, J. S. Pang, D. Ralph, Mathematical Programs with equilibrium Constraints, Cambridge University Press, Cambridge, 1996

[30] C. R. Trujillo, S. Zlobec, Bilevel convex programming models, Optimization 58 (2009), 1009-1028.

[31] Y. Yao, G. Marino L. Muglia, A modified Korpelevich's method convergent to the minimum-norm solution of a variational inequality, Optimization, 63 (2014), 559-569.

[32] D. V. Thong, X-H. Li, Q-L. Dong, Y. J. Cho, T. M. Rassias, A projection and contraction method with adaptive step sizes for solving bilevel pseudomonotone variational inequality problems, Optimization, (2020), DOI:10.1080/02331934.2020.1849206. 
[33] B. Tan, L. Liu, X. Qin, Self adaptive inertial extragradient algorithms for solving bilevel pseudomonotone variational inequality problems, Japan J. Indust. Appl. Math. 38 (2021), 519-543.

[34] T. Kawasaki, W. Takahashi, A strong convergence Theorem for countable families of nonlinear nonself mappings in Hilbert space and application, J. Nonlinear Convex Anal. 19 (2018), 543-560.

[35] P.K. Anh, T.V. Anh, L.D. Muu, On bilevel split pseudomonotone variational inequality problems with applications, Acta. Math. Vietnam 42 (2017), 413-429.

[36] R.W. Cottle, J.C. Yao, Pseudo-monotone complementarity problems in Hilbert space, J. Optim. Theory Appl. 75 (1992), 281-295.

[37] S. Saejung, P. Yotkaew, Approximation of zeros of inverse strongly monotone operators in Banach spaces, Nonlinear Anal. 75 (2012), 742-750.

[38] D. V. Thong, J. Yang, Y. J. Cho, T. M. Rassias, Explicit extragradient-like method with adaptive stepsizes for pseudomonotone variational inequalities, Optim. Lett. 15 (2021), 2181-2199.

[39] J. Yang, H. Liu, Strong convergence result for solving monotone variational inequalities in Hilbert space, Numer. Algo. 80 (2019), 741-752.

[40] H. He, C. Ling, H.K. Xu, A relaxed projection method for split variational inequalities, J. Optim. Theory Appl. 166 (2015), 213-233.

[41] D. V. Thong, P. T. Vuong, Modified Tseng's extragradient methods for solving pseudo-monotone variational inequalities, Optimization, 68 (2019), 2207-2226. 